\title{
49. A STUDY OF O AND B STARS IN VELA, ALONG THE GALACTIC EQUATOR
}

\author{
A. G. VELGHE
}

Koninklijke Sterrenwacht van België, Ukkel, Belgium

The spiral structure of our Galaxy, as defined by optical evidence, shows a striking gap around $l^{\mathrm{II}}=270^{\circ}$. Intrigued by this feature, the author started, in 1955, a survey of early type stars based on objective-prism plates taken with the ADH-Schmidttelescope of the Boyden Observatory (South-Africa); dispersion of the spectra: $240 \AA / \mathrm{mm}$ at $\mathrm{H} \gamma$.

On the basis of the principle of natural groups (Morgan, 1951), 196 OB stars were segregated. The limiting magnitude is 12.5 . For all these stars $U B V$-photometry was carried out with the 60-inch Rockefeller telescope of the Boyden Observatory (1961 and 1962).

The survey deals with the region along the galactic equator, form $l^{\mathrm{II}}=262^{\circ}$ to $273^{\circ}$, and $b^{11}=-4.6$ to $+2^{\circ} .0$. This region shows a conspicuous concentration of early-type stars and several clusters. In earlier work it was supposed to contain the so-called association I Vel, but later on the reality of this association was questioned (Alter et al., 1958). On long-exposure plates (cf: Georgetown Atlas of the Southern Milky Way, 1952), a dark cloud narrows the Milky Way boundaries in this area, and filaments of obscuring matter can be traced within the region concerned.

TABLE I

Visual absorption at a distance of $1.3 \mathrm{kpc}$ for the various regions, indicated by capitals in Figure 1

\begin{tabular}{llll}
\hline Region & $A_{v}$ & Region & $A_{v}$ \\
\hline & & & \\
A & $0^{\mathrm{m}} .45$ & G & $1^{\mathrm{m}} .95$ \\
B & $1^{\mathrm{m}} .15$ & H & $2^{\mathrm{m}} .30$ \\
C & $1^{\mathrm{m}} .30$ & I & $2^{\mathrm{m}} .75$ \\
D & $1^{\mathrm{m}} .35$ & J & $3^{\mathrm{m}} .15$ \\
E & $1^{\mathrm{m}} .50$ & K & $3^{\mathrm{m}} .80$ \\
F & $1^{\mathrm{m}} .95$ & L & $4^{\mathrm{m}} .40$ \\
\hline
\end{tabular}

Figure 1 shows: (a) the surface distribution of the OB stars segregated from the Boyden objective-prism plates; (b) the distribution of the $O$ stars and of those having intrinsic colors $(B-V)_{0}<-0.30$; and (c) the boundaries of the bright starfield and of the dark regions around and within it. Capitals in Figure 1 (c) refer to regions of different obscuration; the corresponding $A_{\downarrow}$-values, given in Table I, are based on data of the stars for which MK types are available (102 stars). 


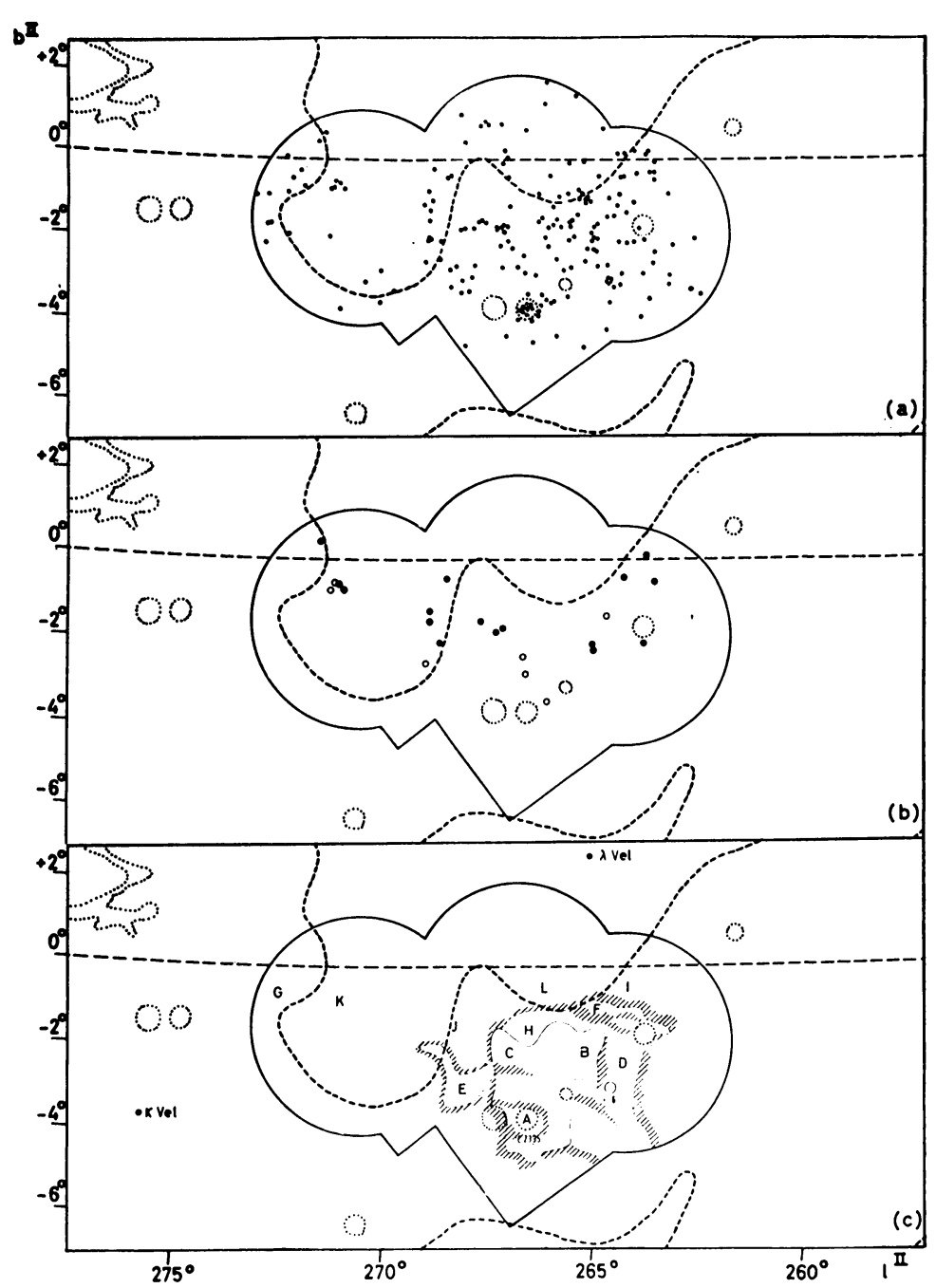

Fig. 1. (a): The surface distribution of the $O B$ stars in the investigated field; (b): the distribution of the $\mathrm{O}$ stars and stars with $(B-V)_{0}<-0.30$; and (c): boundaries of the bright star cloud and of the obscuring matter; capitals indicate specific regions of different absorption as given in Table I. The background material for the drawings is taken from the Skalnate Pléso Atlas of the heavens.

From the discussion of the data appears: (1) that the assumption concerning the reality of an association in the Vela-region has to be rejected; (2) that a spiral feature at right angles to the galactic center extends up to large distances (more than $5 \mathrm{kpc}$ ); this feature could be a linkage between the known Carina-arm and some spiral structure in Puppis, but most of it is hided by obscuring matter. In this connection one should recall that a study of the surface-photometry of the Milky Way by Elsässeand Haug (1960), indicates the existence of an arm in this region, and that the Austrar 
lian radio-observations at $1440 \mathrm{MHz}$ (Mathewson et al., 1962) reveal a not unimportant peak in the same direction. An extension of the present study is dealt with in the paper by Denoyelle, presented at this symposium.

\section{References}

Alter, G., Ruprecht, J., and Vanýsek, V.: 1958, Catalogue of Star Clusters and Associations, Prague.
Elsässer, H. and Haug, U.: 1960, Z. Astrophys. 50, 121.
Mathewson, D. S., Healey, J. R., and Rome, J. M.: 1962, Australian. J. Phys. 15, 354, 369.
Morgan, W. W.: 1951, Publ. Obs. Univ. Michigan 10, 33.
Photographic Atlas of the Southern Milky Way, 1952, Georgetown Coll. Obs. and Nat. Geogr. Soc.,
Washington. 